\title{
Assembly and comparative analysis of the first complete mitochondrial genome of Acer truncatum Bunge: a woody oil-tree species producing nervonic acid
}

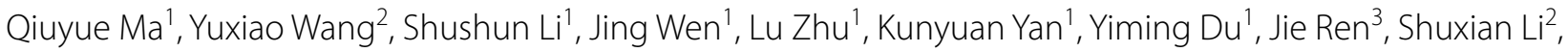
Zhu Chen ${ }^{3}$, Changwei Bi ${ }^{2^{*}}$ and Qianzhong Li $i^{*}$

\begin{abstract}
Background: Acer truncatum (purpleblow maple) is a woody tree species that produces seeds with high levels of valuable fatty acids (especially nervonic acid). The species is admired as a landscape plant with high developmental prospects and scientific research value. The A. truncatum chloroplast genome has recently been reported; however, the mitochondrial genome (mitogenome) is still unexplored.

Results: We characterized the A. truncatum mitogenome, which was assembled using reads from PacBio and Illumina sequencing platforms, performed a comparative analysis against different species of Acer. The circular mitogenome of A. truncatum has a length of 791,052 bp, with a base composition of $27.11 \%$ A, 27.21\% T, 22.79\% G, and 22.89\% C. The A. truncatum mitogenome contains 62 genes, including 35 protein-coding genes, 23 tRNA genes and 4 rRNA genes. We also examined codon usage, sequence repeats, RNA editing and selective pressure in the A. truncatum mitogenome. To determine the evolutionary and taxonomic status of $A$. truncatum, we conducted a phylogenetic analysis based on the mitogenomes of A. truncatum and 25 other taxa. In addition, the gene migration from chloroplast and nuclear genomes to the mitogenome were analyzed. Finally, we developed a novel NAD1 intron indel marker for distinguishing several Acer species.
\end{abstract}

Conclusions: In this study, we assembled and annotated the mitogenome of $A$. truncatum, a woody oil-tree species producing nervonic acid. The results of our analyses provide comprehensive information on the A. truncatum mitogenome, which would facilitate evolutionary research and molecular barcoding in Acer.

Keywords: Acer truncatum, Mitochondrial genome, Repeats, Phylogenetic analysis

\section{Background}

Acer truncatum Bunge (Sapindaceae) is a versatile, oilproducing woody tree widely distributed mainly in northern China, Japan and Korea [1, 2]. This tree species

\footnotetext{
*Correspondence: bichwei@163.com; qianzhongli@jaas.ac.cn ${ }^{1}$ Institute of Leisure Agriculture, Jiangsu Academy of Agricultural Sciences, Nanjing 210014, China

${ }^{2}$ Nanjing Forestry University, Nanjing 210037, China

Full list of author information is available at the end of the article
}

is a potential source of medicinal compounds, including flavonoids, alkaloids, tannins, and terpenoids [3]. Moreover, A. truncatum seed oil contains approximately $90 \%$ unsaturated fatty acids and was listed as a new food resource by the Ministry of Health of the People's Republic of China in 2011 [2]. Nervonic acid (24:15, cis-15-tetracosenoic acid, n-9) accounts for 5-6\% of seed oil [2, 4]. It is a key component of brain nerve cells as well as tissues promoting the repair and regeneration of nerve cells

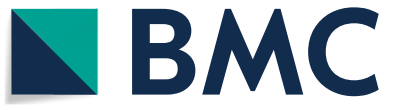

(c) The Author(s) 2022. Open Access This article is licensed under a Creative Commons Attribution 4.0 International License, which permits use, sharing, adaptation, distribution and reproduction in any medium or format, as long as you give appropriate credit to the original author(s) and the source, provide a link to the Creative Commons licence, and indicate if changes were made. The images or other third party material in this article are included in the article's Creative Commons licence, unless indicated otherwise in a credit line to the material. If material is not included in the article's Creative Commons licence and your intended use is not permitted by statutory regulation or exceeds the permitted use, you will need to obtain permission directly from the copyright holder. To view a copy of this licence, visit http://creativecommons.org/licenses/by/4.0/. The Creative Commons Public Domain Dedication waiver (http://creativeco mmons.org/publicdomain/zero/1.0/) applies to the data made available in this article, unless otherwise stated in a credit line to the data. 
and damaged tissues. Previous studies have indicated that nervonic acid is potentially useful for treatment of schizophrenia, psychosis, and attention deficit disorder $[5,6]$. It has been detected in several plant species $[2,7$, 8], but issues related to their nervonic acid content and growth adaptability have limited the utility of these species. The characteristics of rapid growth, wide geographic distribution, and high adaptability, thus $A$. truncatum is a novel potential plant source of nervonic acid for treating human cerebral and neurological problems.

The main function of mitochondria, the "energy factories" of cells, is the conversion of biomass energy into chemical energy in living cells $[9,10]$. In most seed plants, nuclear hereditary information is inherited biparentally, whereas DNA of both mitochondria and chloroplasts is maternally derived $[9,10]$. In addition, recent researches have revealed that intergenomic gene transfer between nuclear and organellar genomes, which was a common phenomenon during plant evolution [11-13]. Along with rapid developments in sequencing and genome assembly technologies, an increasing amount of information on mitogenomes has been uncovered. At present, $6026 \mathrm{com}$ plete land plant organelle genomes, including 5735 chloroplast and 291 plant mitogenomes have been assembled and deposited in GenBank Organelle Genome Resources (https://www.ncbi.nlm.nih.gov/genome/browse/), as the mitochondrial genome is more complex and harder to assemble than that of other organelles $[9,14]$.

Plant mitogenomes are species specific $[15,16]$ and vary considerably in length, gene order, and gene content $[9,10,14,17]$. Genome size is extremely variable, ranging from $66 \mathrm{~kb}$ (Viscum scurruloideum) [18] to $11.3 \mathrm{Mb}$ (Silene conica) [19], and most genomes are $200-800 \mathrm{~kb}$ in size [20]. This wide variation in mitogenome size can be attributed to the repetitive sequences and the foreign DNA from other organisms during evolution [21, 22]. Repetitive sequences, including simple sequence repeats (SSRs), tandem repeats and dispersed repeats, are abundant in the mitogenomes of seed plants. SSRs are frequently used as molecular markers for identifying species in plant mitogenomes [14, 23]. In addition, insertions/ deletions (indels) and single nucleotide polymorphisms (SNPs) within mitogenomes also have been applied to rapidly distinguish species and for phylogenetic analyses $[24,25]$.

The mitochondrial gene content of land plants varies considerably, ranging from 32 to 67 genes. Some genes, including those related to NADH dehydrogenase, ATP synthase, ubiquinol cytochrome, and cytochrome c biogenesis [14], are highly conserved, whereas others, such as $s d h 3, s d h 4$, rps11, and cox2 have been lost [26, 27].

Mitogenomes in the genus Acer, except for the mitogenome sequence of $A$. yangbiense released in 2019, have not been analyzed in detail [28]. In this study, we first assembled the complete mitogenome of A. truncatum and analyzed its gene content, repetitive sequences, RNA editing sites, selective pressure, and phylogenetic relationships. We also surveyed gene transfer among nuclear, chloroplast, and mitochondrial genomes of A. truncatum. Moreover, we developed a marker based on an indel in the NAD1 intron to distinguish seven Acer species (A. buergerianum, A. truncatum, A. henryi, A. negundo, A. ginnala, A. yangbiense and A. tonkinense). The data presented herein expand genetic information available for the genus Acer and provide an opportunity to conduct further important genomic breeding studies on $A$. truncatum.

\section{Results}

\section{Features of the $A$. truncatum mitogenome}

The $A$. truncatum genome sequence generated was submitted to the GenBank database (accession number MZ318049) in this study. The complete mitogenome of A. truncatum is $791,052 \mathrm{bp}$ in length and has the typical circular structure of land plant genomes (Fig. 1). The nucleotide composition of the complete mitogenome is 27.11\% A, 27.21\% T, 22.79\% G, and 22.89\% C, with a GC content of $45.68 \%$ (Table 1). Protein-coding genes (PCGs) and cis introns account for 4.31 and $2.94 \%$ of the whole mitogenome, while tRNA and rRNA genes comprise only 0.22 and $0.67 \%$, respectively. A total of 62 unique genes, including 35 protein-coding, 23 tRNA, and 4 rRNA genes, were identified in the A. truncatum mitogenome (Table 2). Interestingly, two copies of cox 1 genes were found. Additionally, five tRNA and one rRNA gene(s) located in repeat sequences were found to be present in two or four copies (trnN-GTT, trnM-CAT, trnP-TGG, trnH-GTG, trn W-CCA, and rrn5) (Fig. 1).

\section{Codon usage analysis of PCGs}

The total length of PCGs in A. truncatum was 34,059bp. Most PCGs had the typical ATG start codon, whereas atp6, nad1, and nad4L had ACG as the start codonpresumably a consequence of C-to-U RNA editing of the second site (Table 2). Three types of stop codons were identified, namely, TAA, TGA, and TAG, the C to U RNA editing phenomenon was not found in the stop codons. As shown in Fig. 2, the codon usage analysis revealed the most frequent amino acids to be leucine (Leu) (11.2$11.3 \%)$, serine (Ser) (10.6-11.0\%), and arginase (Arg) (8.1-8.4\%), whereas cysteine (Cys) and tryptophan (Trp) were rarely found.

We also analyzed the relative synonymous codon usage (RSCU) of 35 PCGs in the A. truncatum mitogenome. As shown in Fig. 3, the 35 PCGs comprised 


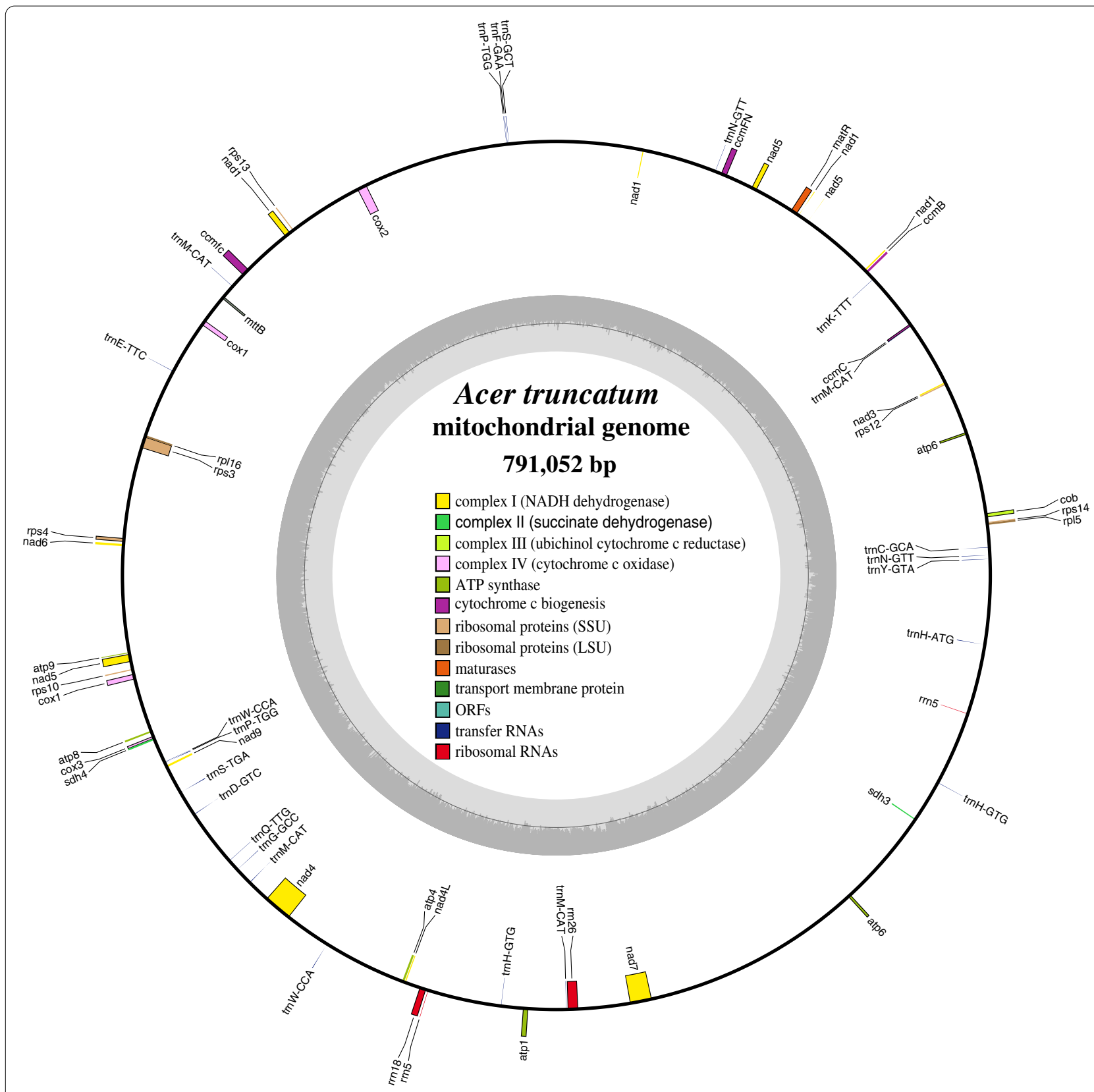

Fig. 1 Circular map of the A. truncatum mitogenome. Genes shown on the outside and inside of the circle are transcribed clockwise and counterclockwise, respectively. The dark gray region in the inner circle depicts GC content. Asterisks besides genes denote intron-containing genes

33,948 bp encoding 11,316 codons excluding termination codons. We found that nearly all of the RSCU values of NNT and NNA codons were higher than 1.0 with the exception of Ile (AUA, 0.82), Leu (CUA, 0.93), and Ser (UCA, 0.97). Codon usage was generally strongly biased toward $\mathrm{A}$ or $\mathrm{T}(\mathrm{U})$ at the third codon position in the A. truncatum mitogenome, which is very common in mitogenomes of land plant species.

\section{Analysis of synonymous and nonsynonymous substitution} rates

In genetics, the nonsynonymous-to-synonymous substitution ratio $(\mathrm{Ka} / \mathrm{Ks})$ is used to understand the evolutionary dynamics of genes. In this study, the $\mathrm{Ka} / \mathrm{Ks}$ ratio was determined for 26 protein-coding genes common to A. truncatum, A. yangbiense, A. thaliana and C. sinensis mitogenomes (Fig. 4). The PCGs shared between A. truncatum and $A$. yangbiense were close homologs, as the $\mathrm{Ka} /$ 
Table 1 Genomic features of the A. truncatum mitogenome

\begin{tabular}{|c|c|c|c|c|c|c|c|}
\hline Feature & $A(\%)$ & $C(\%)$ & $G(\%)$ & $\mathrm{T}(\%)$ & GC(\%) & Size (bp) & $\begin{array}{l}\text { Proportion } \\
\text { in Genome } \\
(\%)\end{array}$ \\
\hline Whole genome & 27.11 & 22.89 & 22.79 & 27.21 & 45.68 & 791,052 & 100 \\
\hline Protein-coding genes & 26.12 & 31.1 & 21.52 & 21.25 & 52.62 & 34,059 & 4.31 \\
\hline cis-spliced introns & 23.59 & 26.56 & 26.56 & 24.76 & 53.11 & 23,222 & 2.94 \\
\hline tRNA genes & 24.59 & 26.27 & 24.77 & 24.36 & 51.04 & 1728 & 0.22 \\
\hline rRNA genes & 23.37 & 26.34 & 25.74 & 24.55 & 52.08 & 5280 & 0.67 \\
\hline Non-coding regions & 27.30 & 22.36 & 22.36 & 27.60 & 44.71 & 72,6763 & 91.87 \\
\hline
\end{tabular}

$K s$ ratio of 21 PCGs was 0 . In addition, nearly all $\mathrm{Ka} / \mathrm{Ks}$ ratios were less than 1.0, which suggested that most of the PCGs were subject to stabilizing selection during evolution. Conversely, the $\mathrm{Ka} / \mathrm{Ks}$ ratios of nine genes (atp6, cob, cox1, nad2, ccmFn, nad4, nad6, nad7 and rpl5) were greater than 1.0, which indicateed these genes had been under positive selection during evolution. Finally, three genes (atp4, ccmB and rps4) had $K a / K s$ ratios close to 1 , thus suggested that they had experienced neutral evolution since the divergence of their common ancestor.

\section{Prediction of RNA editing sites in PCGs}

In plants, RNA editing is necessary for gene expression, with cytidine (C)-to-uridine (U) RNA editing enriched in mitochondrial and chloroplast genomes. In this study, we predicted the RNA editing sites of 26 PCGs common to mitogenomes of four angiosperm species. The number of RNA editing sites predicted for A. truncatum, A. yangbiense, A. thaliana, and C. sinensis-421, 427, 342 and 288, respectively-suggests that these sites are extremely conserved in PCGs in Acer. A total of 421 RNA editing sites were predicted in $A$. truncatum, all exhibiting C-toU RNA editing. Among the 421 sites, 32.07 and 67.93\% were predicted at the first and the second positions of codons, respectively, whereas none were found at the third position (Fig. 5).

RNA editing can change PCG initiation and termination codons. As shown in Table 2, atp6, nad1 and nad4L genes use ACG as their initiation codons, we infer that they may have been altered by RNA editing. The number of RNA editing sites in different genes was found to vary greatly, with the largest predicted numbers detected in cytochrome $c$ biogenesis $(c c m B, c c m C, c c m F n$, and ccmFc), Complex I (NADH dehydrogenase) and nad4 genes. In contrast, no RNA editing sites were found in atp 9 and nad 3 genes in A. truncatum and A. yangbiense.

\section{Analysis of repeats in the $A$. truncatum mitogenome}

An analysis of repeats in the A. truncatum mitogenome revealed 503 long repeats (> $30 \mathrm{bp}$ ), namely, 287 forward
(57.05\%), 179 palindromic (35.59\%), 33 reverse $(6.60 \%)$ and 1 complementary $(0.20 \%)$ repeats (Fig. 6A). The total length of the long repeats was $144,318 \mathrm{bp}$, which corresponded to $18.24 \%$ of the mitogenome. Most repeats were $35-50$ bp long (254 repeats, $50.29 \%$ ), whereas 24 were longer than $1 \mathrm{~kb}$, the largest was $28,452 \mathrm{bp}$ (Fig. 6B and Table S1). In A. truncatum mitogenome, we found that five pair of large repeats $(>1 \mathrm{~kb})$ by rearrangements could produce two subgenomic circles, which comprising of $457,840 \mathrm{bp}$ and $333,212 \mathrm{bp}$, mediated by the pairwise large repeats R3a and R3b (Table S2 and Fig.S1). We also identified repeats in the A. yangbiense mitogenome to further characterize repeats in Acer species. A total of 500 long repeats were found, including 271 forward (54.20\%), 88 palindromic (17.60\%), and 141 reverse (28.20\%) repeats. No complementary repeats were identified. The total length constituted by long repeats was $138,024 \mathrm{bp}$, which accounted for $17.18 \%$ of the A. yangbiense mitogenome (803, $281 \mathrm{bp}$ ) (Fig. S2A and B). Most repeats were $41-60 \mathrm{bp}$ long (288 repeats, $57.60 \%$ ), the longest repeat was 27,124bp (Table S3).

SSRs, which are tandem repeated sequences with motifs of one to six bases, are useful molecular markers for studying genetic diversity and identifying species $[14,23]$. In this study, a total of 717 SSRs were detected in the A. truncatum mitogenome, including 226 (31.52\%) mono-, 355 (49.51\%) di-, 49 (6.83\%) tri-, 67 (9.34\%) tetra-, $18(2.51 \%)$ penta-, and $2(0.28 \%)$ hexanucleotide repeats (Table 3). Among the 717 SSRs, more than $81 \%$ were mono- and di-repeats. Further analysis of SSR repeat units indicated that $85.40 \%$ of monomers had $\mathrm{A} / \mathrm{T}$ contents, and $45.07 \%$ of dinucleotide repeats were AT/TA. The higher AT content of SSRs contributed to the AT richness $(54.32 \%)$ of the complete A. truncatum mitogenome.

\section{Phylogenetic analysis}

To determine the phylogenetic position of A. truncatum, we downloaded 25 plant mitogenomes from GenBank (https://www.ncbi.nlm.nih.gov/genome/browse/) (Table 
Table 2 Gene profile and organization of the A. truncatum mitogenome

\begin{tabular}{|c|c|c|c|c|c|}
\hline Group of genes & Gene name & Length & Start codon & Stop codon & Amino acids \\
\hline \multirow[t]{5}{*}{ ATP synthase } & atp1 & 1530 & ATG & TGA & 509 \\
\hline & atp4 & 597 & ATG & TAG & 198 \\
\hline & atp6 & 774 & ACG & TAA & 257 \\
\hline & atp8 & 480 & ATG & TAA & 159 \\
\hline & atp9 & 225 & ATG & TGA & 74 \\
\hline \multirow[t]{9}{*}{ NADH dehydrogenase } & $\operatorname{nad}^{a}$ & 978 & ACG & TAA & 325 \\
\hline & $\operatorname{nad} 2^{a}$ & 1467 & ATG & TAA & 488 \\
\hline & nad3 & 357 & ATG & TAA & 118 \\
\hline & nad $4^{a}$ & 1488 & ATG & TGA & 495 \\
\hline & nad4l & 303 & ACT & TAA & 100 \\
\hline & nad5 & 2004 & ATG & TAA & 667 \\
\hline & nad6 & 618 & ATG & TAA & 205 \\
\hline & $\operatorname{nad} 7^{a}$ & 1185 & ATG & TAG & 394 \\
\hline & nad9 & 573 & ATG & TAA & 190 \\
\hline \multirow[t]{4}{*}{ Cytochrome $\mathrm{c}$ biogenesis } & $\mathrm{ccmB}$ & 621 & ATG & TGA & 206 \\
\hline & $\mathrm{ccmC}$ & 753 & ATG & TGA & 250 \\
\hline & $c \mathrm{cmF} \mathrm{C}^{a}$ & 1365 & ATG & TAG & 454 \\
\hline & $\mathrm{ccmFn}$ & 1734 & ATG & TGA & 577 \\
\hline Maturases & matR & 1962 & ATG & TAG & 653 \\
\hline Ubichinol cytochrome c reductase & $c o b$ & 1182 & ATG & TGA & 393 \\
\hline \multirow[t]{3}{*}{ Cytochrome c oxidase } & $\operatorname{cox} 1(2)$ & 1584 & ATG & TAA & 527 \\
\hline & $\cos 2$ & 795 & ATG & TGA & 264 \\
\hline & $\cos 3$ & 798 & ATG & TGA & 265 \\
\hline Transport membrane protein & $m t t B$ & 792 & ATA & TAG & 264 \\
\hline \multirow[t]{2}{*}{ Ribosomal proteins (LSU) } & $r p / 5$ & 555 & ATG & TAA & 184 \\
\hline & $r p / 16$ & 516 & ATG & TAA & 171 \\
\hline \multirow[t]{6}{*}{ Ribosomal proteins (SSU) } & $r p s 3^{a}$ & 1686 & ATG & TAA & 561 \\
\hline & $r p s 4$ & 1077 & ATG & TAA & 358 \\
\hline & $\operatorname{rps} 10^{\circ}$ & 330 & ATG & TAA & 109 \\
\hline & $r p s 12$ & 378 & ATG & TGA & 125 \\
\hline & $r p s 13$ & 294 & ATG & TGA & 97 \\
\hline & $\operatorname{rps} 14$ & 255 & ATG & TGA & 84 \\
\hline \multirow[t]{2}{*}{ Succinate dehydrogenase } & $\operatorname{sch} 3$ & 327 & ATG & TGA & 108 \\
\hline & $\operatorname{sdh} 4$ & 480 & ATG & TAA & 159 \\
\hline \multirow[t]{16}{*}{ Transfer RNAs } & $\operatorname{trnY-GTA}$ & 83 & - & - & - \\
\hline & $\operatorname{trnN}-G T^{b}(2)$ & 72 & - & - & - \\
\hline & $\operatorname{trnC}-G C A$ & 71 & - & - & - \\
\hline & $\operatorname{trnM}-\mathrm{CAT}(4)$ & $73 / 74 / 74 / 77$ & - & - & - \\
\hline & trnK-TTT & 73 & - & - & - \\
\hline & $\operatorname{trnS}-G C T$ & 88 & - & - & - \\
\hline & $\operatorname{trnF-GAA}$ & 74 & - & - & - \\
\hline & $\operatorname{trn} P-T G G^{b}(2)$ & $74 / 75$ & - & - & - \\
\hline & $\operatorname{trnE-TTC}$ & 72 & - & - & - \\
\hline & $\operatorname{trn} W-C C A^{b}(2)$ & $73 / 74$ & - & - & - \\
\hline & $\operatorname{trnS}-T G A$ & 87 & - & - & - \\
\hline & $\operatorname{trnD}-G T C^{b}$ & 74 & - & - & - \\
\hline & $\operatorname{trnQ} Q-T T G$ & 72 & - & - & - \\
\hline & $\operatorname{trnG-GCC}$ & 72 & - & - & - \\
\hline & $\operatorname{trn} H-G T G^{b}(2)$ & $74 / 74$ & - & - & - \\
\hline & $\operatorname{trnH}-A T G$ & 76 & - & - & - \\
\hline \multirow[t]{3}{*}{ Ribosomal RNAs } & $\operatorname{rrn5}(2)$ & $119 / 120$ & - & - & - \\
\hline & $r m 18$ & 1939 & - & - & - \\
\hline & $r m 26$ & 3102 & - & - & - \\
\hline
\end{tabular}

Note: Numbers after gene names are the number of copies. The superscripts a and b indicate genes containing introns and chloroplast-derived genes, respectively 


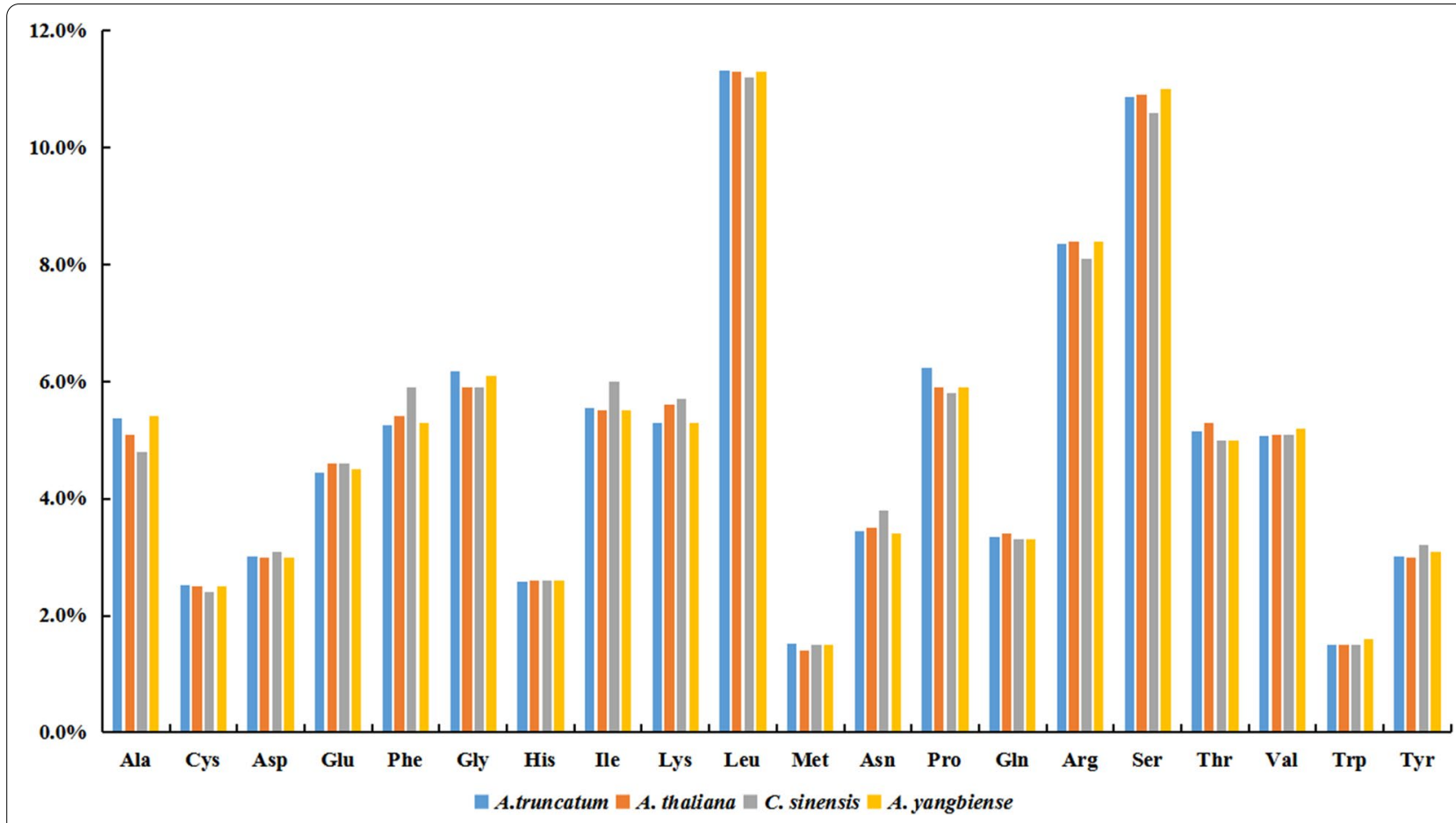

Fig. 2 Codon usage pattern of the A. truncatum mitogenome compared with A. yangbiense, A. thaliana, and C. sinensis. The relative percentage of each amino acid residue in all mitochondrial proteins is shown on the $y$-axis

S4) and constructed a phylogenetic tree based on a set of 25 conserved single-copy orthologous genes present in all 26 analyzed mitogenomes. As shown in Fig. 7, 21 of 23 nodes in the generated tree had bootstrap support values over $70 \%$, including 12 nodes with $100 \%$ support. The phylogenetic tree strongly supports $(100 \%$ bootstrap support) the close phylogenetic relationship between $A$. truncatum and A. yangbiense. In addition, it also revealed that both species were closely related to $C$. sinensis, which is similar to conclusions inferred using the nuclear genome [2]. Overall, the results of our analysis of mitogenomes provide a valuable foundation for future analyses of the phylogenetic affinities of Acer species.

\section{Plastid-derived and nuclear-shared sequence transfer events}

DNA fragment transfers among nuclear and organellar genomes are common events during plant evolution. Six directions of gene transfer are possible among the three types of genomes. To further understand the characteristics of sequence transfer events in A. truncatum, the $A$. truncatum nuclear and chloroplast genomes [2, 29] were searched by using its mitogenome sequences as queries. We obtained 393 hits covering $230.0 \mathrm{~kb}$ of sequences of nuclear genome transferred into the mitogenome. According to the nuclear-mitochondrial alignment, hits occurred on every A. truncatum chromosome (Fig. 8A), however, the total lengths of the hits and the percent coverage on the chromosomes were different. Chromosome 1 had the maximum total length of hits $(25.30 \mathrm{~kb})$, which was much larger than on other chromosomes, whereas the highest percent coverage $(0.05 \%)$ occurred on chromosomes 5,6 , and 13 . In addition, fragment lengths were mainly between $200 \mathrm{bp}$ and $400 \mathrm{bp}$ (Fig. 8B). A total of $62,241 \mathrm{bp}$ of sequences $(7.87 \%$ of the A. truncatum mitogenome) were found to be shared between nuclear and mitochondrial genomes. The shared sequences contained seven complete genes (trnN-GTT , rpl5, trnS-GCT, trnF-GAA, trnQ-TTG, atp1, and trnHGTG) as well as partial gene sequences of matR, $c c m F N$, cox2, rps3, rps4, atp8, sdh4, nad4 and atp6.

The A. truncatum mitogenome sequence (791,052bp) was approximately five times longer than the chloroplast genome $(156,492 \mathrm{bp})$. Forty-one fragments with a total length of $18,637 \mathrm{bp}$, corresponding to $2.36 \%$ of the mitogenome, were observed to have migrated from the chloroplast genome to the mitogenome in A. truncatum (Table 4). Six intact chloroplast genes (psbJ, trnP-UGG, trn W-CCA, trnN-GUU, trnD-GUC, and trnH-GUG) were located on these fragments. The remaining fragments were partial sequences of transferred genes or intergenic spacer regions in the chloroplast genome. Interestingly, we found 


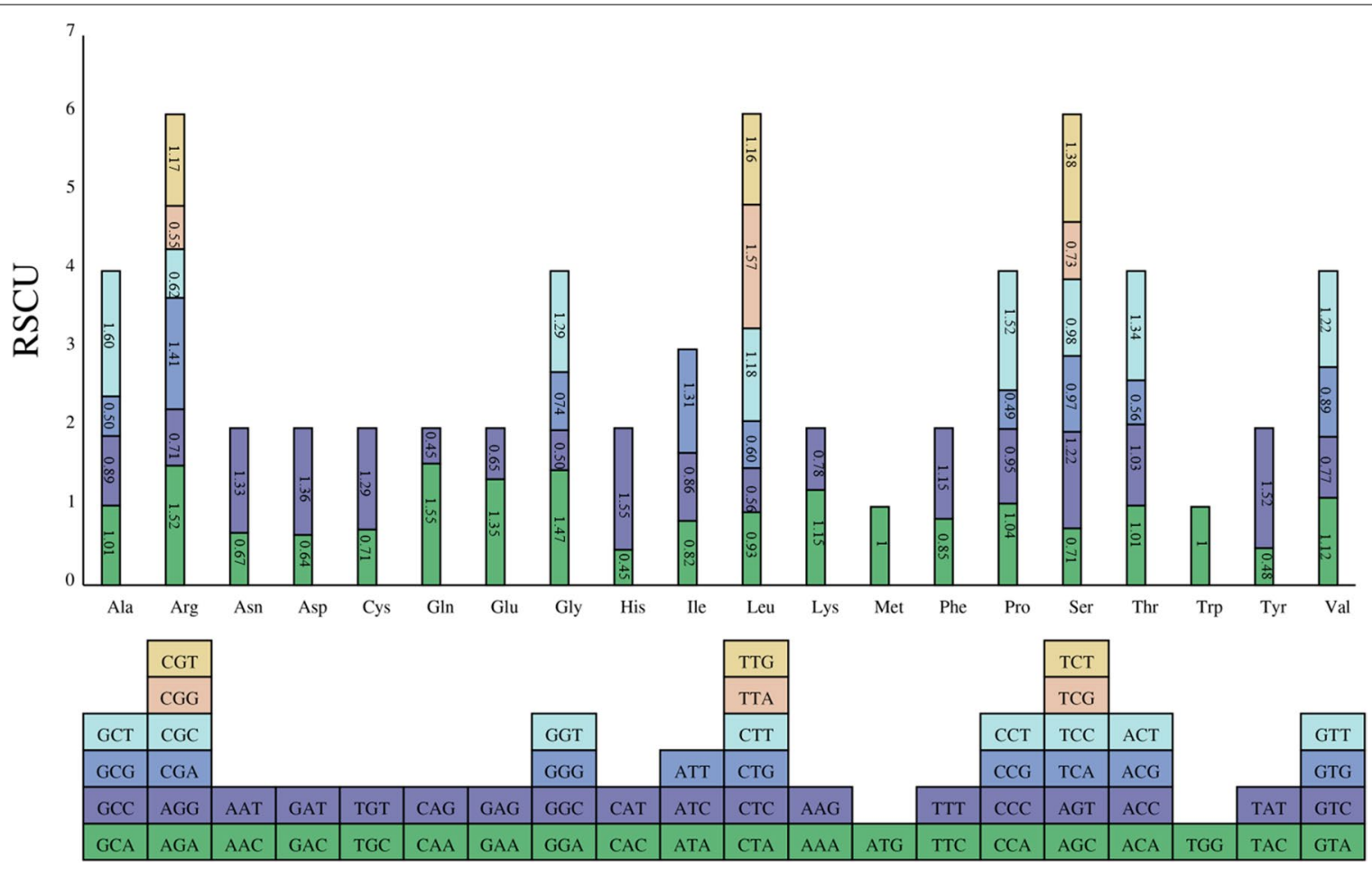

Fig. 3 Relative synonymous codon usage (RSCU) in the A. truncatum mitogenome. Codon families are shown on the $x$-axis. RSCU values are the number of times a particular codon is observed relative to the number of times that codon would be expected for a uniform synonymous codon usage

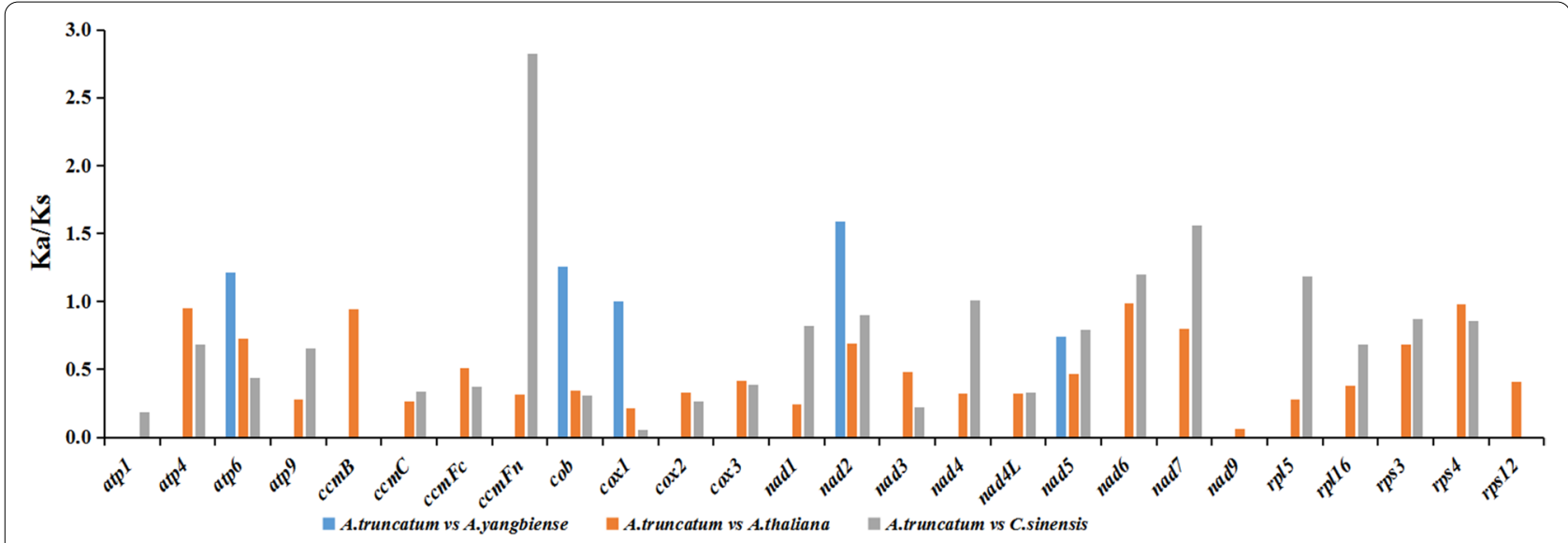

Fig. $4 \mathrm{Ka} / \mathrm{Ks}$ ratios of 26 protein-coding genes in A. truncatum, A. yangbiense, A. thaliana, and C. sinensis

that the DNA migration had often occurred in the inverted repeat region of the $A$. truncatum chloroplast genome.

\section{Development of an NAD1 intron indel marker}

Among Acer species, only the mitogenome of A. yangbiense has currently been reported. To further characterize the NAD1 intron, we compared its sequence between $A$. truncatum and A. yangbiense, and detected a 33-bp indel. The following seven Acer species were selected for characterization of the NAD1 intron sequence: $A$. truncatum, $A$. buergerianum, $A$. ginnala, A. yangbiense, A. palmatum, A. pubipalmatum, and A. tonkinense. To develop indel markers, primers were designed to anneal to conserved regions 


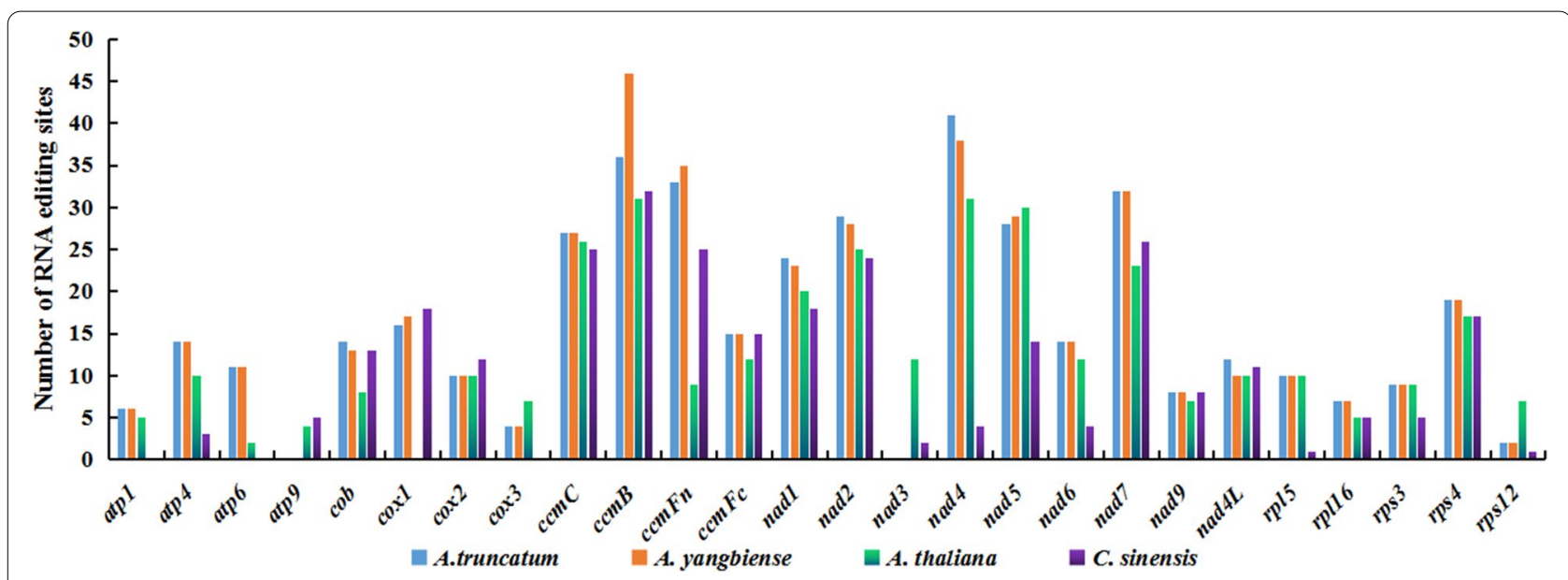

Fig. 5 The distribution of RNA editing sites in mitogenome protein-coding genes of four angiosperms
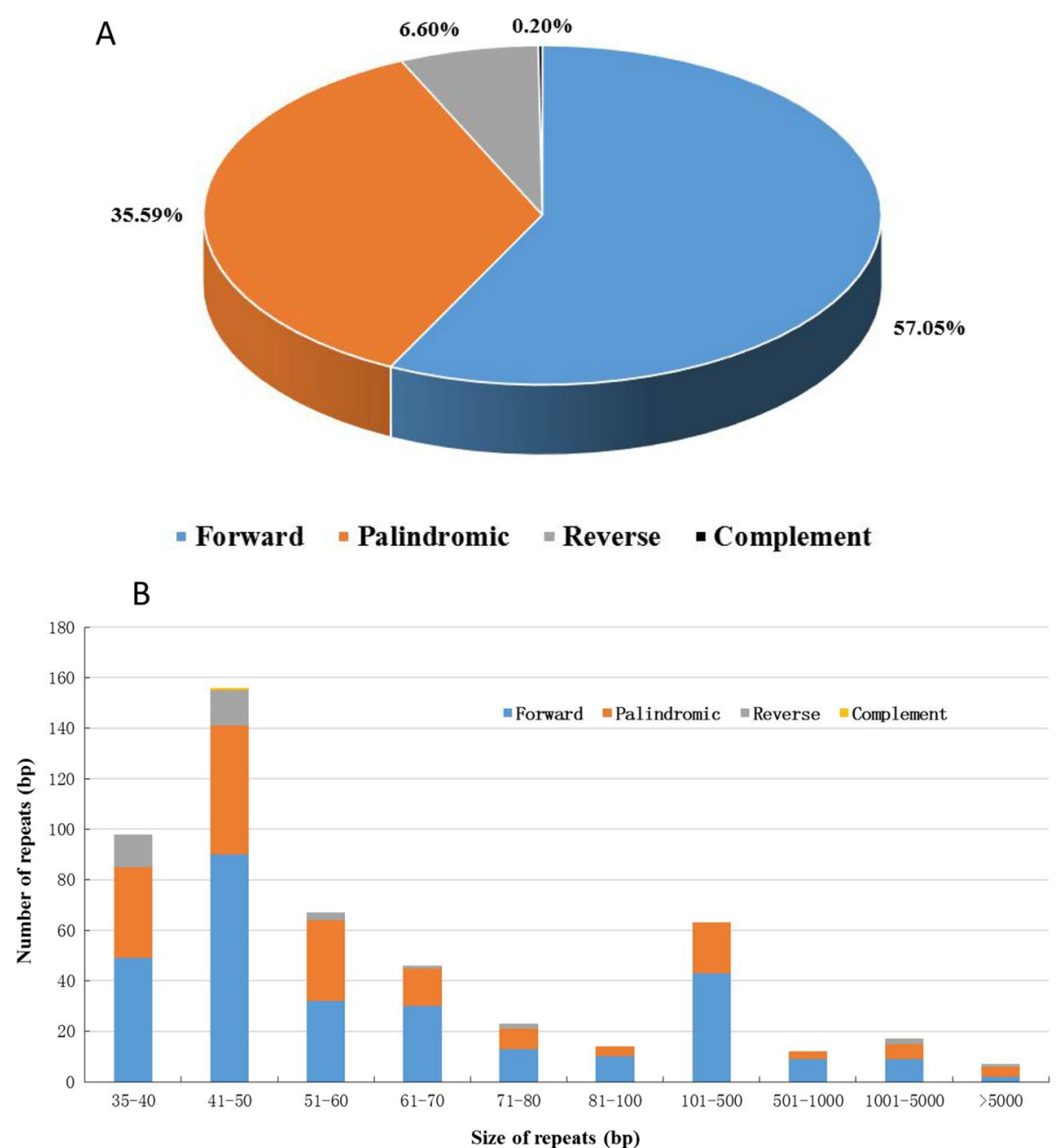

Fig. 6 Detected repeats in the A. truncatum mitogenome. A Type and proportion of detected repeats. B Frequency distribution of repeat lengths 
Table 3 Frequency of identified SSR motifs in the A. truncatum mitogenome

\begin{tabular}{|c|c|c|c|c|c|c|c|c|c|c|c|c|c|c|c|}
\hline \multirow[t]{2}{*}{ Motif Type } & \multicolumn{13}{|c|}{ Number of repeats } & \multirow[t]{2}{*}{ Total } & \multirow[t]{2}{*}{ Proportion (\% } \\
\hline & 3 & 4 & 5 & 6 & 7 & 8 & 9 & 10 & 11 & 12 & 14 & 15 & 21 & & \\
\hline Monomer & - & - & - & - & - & 118 & 65 & 28 & 7 & 3 & 2 & 2 & 1 & 226 & 31.52 \\
\hline Dimer & - & 280 & 51 & 14 & 5 & 2 & 2 & 1 & - & - & - & - & - & 355 & 49.51 \\
\hline Trimer & - & 43 & 3 & 1 & 1 & & 1 & - & - & - & - & - & - & 49 & 6.83 \\
\hline Tetramer & 59 & 7 & 1 & - & - & - & - & - & - & - & - & - & - & 67 & 9.34 \\
\hline Pentamer & 16 & - & - & 2 & - & - & - & - & - & - & - & - & - & 18 & 2.51 \\
\hline Hexamer & 2 & - & - & - & - & - & - & - & - & - & - & - & - & 2 & 0.28 \\
\hline Total & 77 & 330 & 55 & 17 & 6 & 120 & 68 & 29 & 7 & 3 & 2 & 2 & 1 & 717 & 100 \\
\hline
\end{tabular}

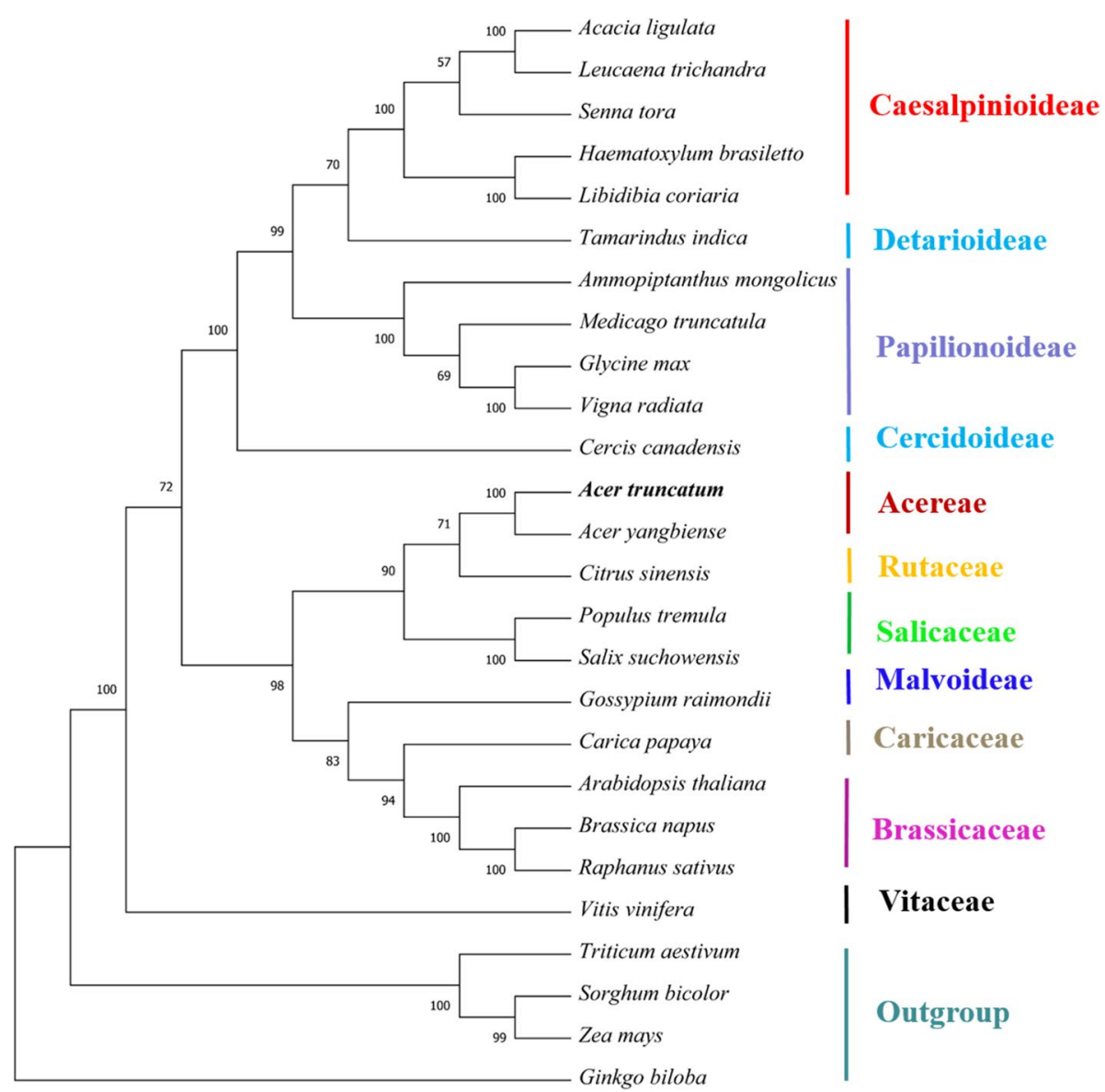

Fig. 7 Maximum-likelihood phylogenetic tree based on 25 single-copy orthologous genes shared among 26 species. Numbers at nodes are bootstrap support values. The position of A. truncatum is indicated in bold. Triticum aestivum, Sorghum bicolor, Ginkgo biloba, and Zea mays served as outgroups

of the NAD1 intron (Table S5). The predicted amplification products were successfully obtained using these NAD1-intron-F/R primers in all seven tested samples (Fig. 9A). In all six species, the length of the amplified NAD1 intron sequence was identical
(808 bp) and highly conserved. The corresponding sequence in $A$. yangbiense was indeed longer ( $841 \mathrm{bp}$ ) because of the 33-bp putative insertion (Fig. 9A and B). Several species close to A. truncatum in the phylogenetic tree (A. yangbiense, Populus tremula, Salix suchowensis and $C$. sinensis) were selected to verify 


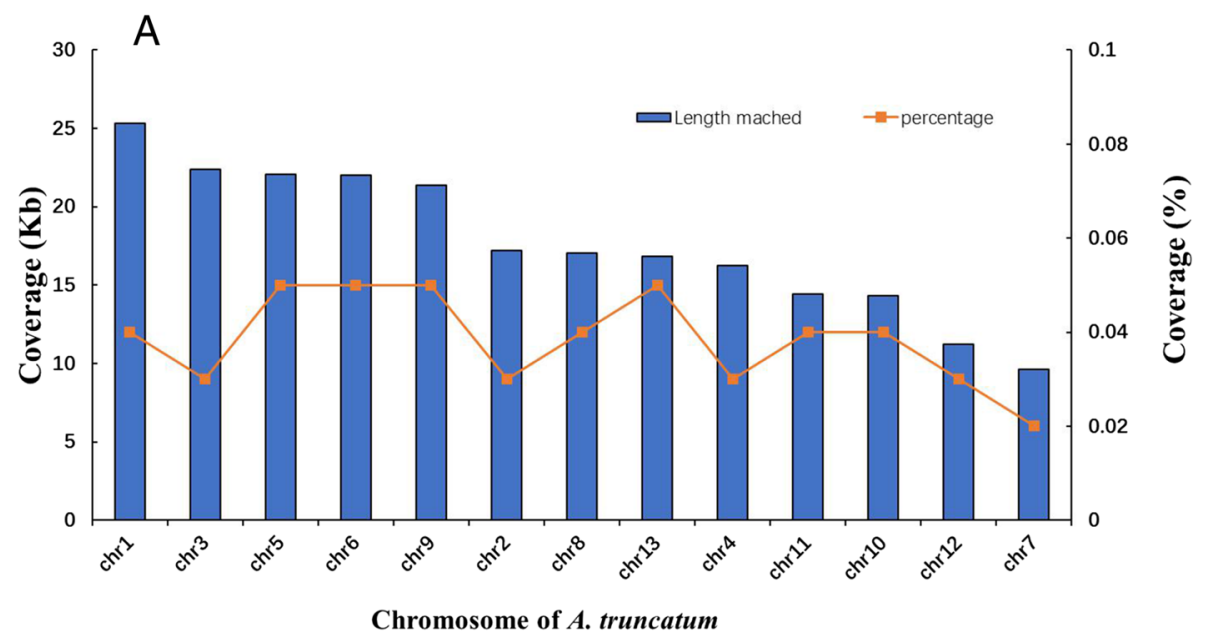

B

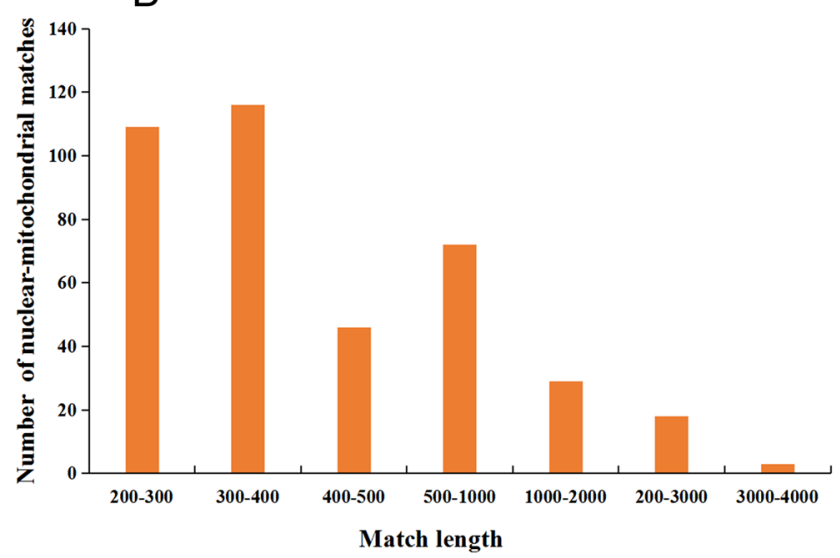

Fig. 8 Characteristics of nuclear-mitochondrial sequences in A. truncatum. A Distributions of percent identities between shared nuclearmitochondrial matches. The number of matches is shown by blue boxes and is plotted on the left ordinate. The orange lines, which represent the coverage of matches on nuclear and mitochondrial genomes, are plotted on the right ordinate. B Distributions of lengths between shared nuclearmitochondrial matches

whether the 33-bp sequence was an insertion or a deletion. According to the sequence alignment, the sequence was indeed an insertion (Fig. S3). In previous studies, indel markers have frequently been used to distinguish closely related species; however, Acer species have not been identified on the basis of their mitogenomes using this approach. Our firstever characterization of the NAD1 intron in Acer may therefore be applicable for classification and identification of Acer species.

\section{Discussion}

\section{Characterization of the A. truncatum mitochondrial genome}

Mitochondria, which produce the energy required to carry out life processes, are the powerhouses of plants. Because of factors such as size variation and repeated sequences, plant mitogenomes are more complex than those of animals $[9,14,30,31]$. The emergence of rapid, cost-effective genome sequencing technologies has accelerated understanding of mitogenomes. Our study has produced the first detailed characterization of a complete mitogenome in Acer. The size of the A. truncatum mitogenome is similar to that of $A$. yangbiense [28], both of which are moderate in size relative to most genomes [32]. GC content is an important factor for assessing species. The GC content of the A. truncatum mitogenome is $45.68 \%$, which is comparable to that of other sequenced plant mitogenomes (A. thaliana, 44.8\% [33]; Phaseolus vulgaris, 45.11\% [14]; Beta vulgaris, 43.9\% [34], but higher than the A. truncatum chloroplast genome (37.90\%) assembled by our research group [29]. Similar to most other mitogenomes, most sequences in the $A$. truncatum mitogenome are non-coding. Protein-coding 
Table 4 Fragments transferred from chloroplasts to mitochondria in A. truncatum

\begin{tabular}{|c|c|c|c|c|c|c|c|c|c|}
\hline & $\begin{array}{l}\text { Alignment } \\
\text { Length }\end{array}$ & $\begin{array}{l}\text { Identity } \\
\%\end{array}$ & Mismatch & Gap opens & CP Start & CP End & Mt Start & Mt End & Gene \\
\hline 1 & 2890 & 99.689 & 9 & 0 & 22,796 & 25,685 & 588,995 & 586,106 & rpoCl \\
\hline 2 & 2890 & 99.239 & 21 & 1 & 22,796 & 25,685 & 762,940 & 760,052 & rpoCl \\
\hline 3 & 2700 & 99.963 & 1 & 0 & 20,067 & 22,766 & 699,997 & 697,298 & \\
\hline 4 & 1259 & 97.935 & 7 & 8 & 99,134 & 100,376 & 576,186 & 574,931 & \\
\hline 5 & 1259 & 97.935 & 7 & 8 & 142,127 & 143,369 & 574,931 & 576,186 & \\
\hline 6 & 1259 & 97.935 & 6 & 9 & 142,127 & 143,369 & 730,678 & 729,424 & \\
\hline 7 & 1259 & 97.935 & 6 & 9 & 99,134 & 100,376 & 729,424 & 730,678 & \\
\hline 8 & 1067 & 90.909 & 55 & 28 & 65,889 & 66,926 & 449,842 & 450,895 & psbJ \\
\hline 9 & 351 & 99.715 & 1 & 0 & 45,259 & 45,609 & 437,233 & 437,583 & $y c f 3$ \\
\hline 10 & 349 & 99.713 & 1 & 0 & 75,224 & 75,572 & 46,646 & 46,994 & $p s b B$ \\
\hline 11 & 224 & 99.107 & 1 & 1 & 138,184 & 138,407 & 589,548 & 589,326 & $\operatorname{trn} l-G A U$ \\
\hline 12 & 224 & 99.107 & 1 & 1 & 104,096 & 104,319 & 589,326 & 589,548 & trnl-GAU \\
\hline 13 & 205 & 93.171 & 12 & 2 & 66,231 & 66,434 & 362,782 & 362,579 & $p s b F$ \\
\hline 14 & 173 & 94.798 & 9 & 0 & 68,227 & 68,399 & 451,672 & 451,844 & $\operatorname{trn} P-U G G$ \\
\hline 15 & 141 & 100 & 0 & 0 & 110,171 & 110,311 & 150,810 & 150,670 & \\
\hline 16 & 141 & 100 & 0 & 0 & 132,192 & 132,332 & 150,670 & 150,810 & \\
\hline 17 & 131 & 100 & 0 & 0 & 35,802 & 35,932 & 277,631 & 277,761 & $p s b C$ \\
\hline 18 & 123 & 92.683 & 9 & 0 & 67,987 & 68,109 & 451,461 & 451,583 & $\operatorname{trn} W-C C A$ \\
\hline 19 & 93 & 100 & 0 & 0 & 136,588 & 136,680 & 694,605 & 694,513 & $\operatorname{trn} A-U G C$ \\
\hline 20 & 93 & 100 & 0 & 0 & 105,823 & 105,915 & 694,513 & 694,605 & $\operatorname{trn} A-U G C$ \\
\hline 21 & 98 & 97.959 & 2 & 0 & 110,389 & 110,486 & 150,666 & 150,569 & \\
\hline 22 & 98 & 97.959 & 2 & 0 & 132,017 & 132,114 & 150,569 & 150,666 & $\operatorname{trnN}-G \cup U$ \\
\hline 23 & 178 & 84.831 & 17 & 8 & 30,780 & 30,955 & 468,861 & 469,030 & $\operatorname{trnD}-G \cup C$ \\
\hline 24 & 105 & 96.19 & 3 & 1 & 109,900 & 110,003 & 533,208 & 533,104 & \\
\hline 25 & 105 & 96.19 & 3 & 1 & 132,500 & 132,603 & 533,104 & 533,208 & \\
\hline 26 & 90 & 98.889 & 1 & 0 & 151,306 & 151,395 & 452,466 & 452,377 & $y c f 2$ \\
\hline 27 & 90 & 98.889 & 1 & 0 & 91,108 & 91,197 & 452,377 & 452,466 & $y c f 2$ \\
\hline 28 & 83 & 100 & 0 & 0 & 59,800 & 59,882 & 72,900 & 72,982 & $a c c D$ \\
\hline 29 & 86 & 96.512 & 3 & 0 & 132,009 & 132,094 & 5982 & 5897 & $\operatorname{trnN}-G U U$ \\
\hline 30 & 86 & 96.512 & 3 & 0 & 110,409 & 110,494 & 5897 & 5982 & $\operatorname{trnN}-G U U$ \\
\hline 31 & 79 & 98.734 & 1 & 0 & 54,466 & 54,544 & 494,502 & 494,424 & $\operatorname{trnM}-C A U$ \\
\hline 32 & 75 & 100 & 0 & 0 & 154,389 & 154,463 & 77,164 & 77,090 & $\operatorname{trn} l-C A U$ \\
\hline 33 & 75 & 100 & 0 & 0 & 88,040 & 88,114 & 77,090 & 77,164 & $\operatorname{trnl}-\mathrm{CAU}$ \\
\hline 34 & 80 & 97.5 & 2 & 0 & 7 & 86 & 577,140 & 577,219 & $\operatorname{trnH}-G \cup G$ \\
\hline 35 & 80 & 97.5 & 2 & 0 & 7 & 86 & 728,470 & 728,391 & $\operatorname{trnH}-G \cup G$ \\
\hline 36 & 72 & 100 & 0 & 0 & 101,734 & 101,805 & 206,176 & 206,105 & \\
\hline 37 & 72 & 100 & 0 & 0 & 140,698 & 140,769 & 206,105 & 206,176 & \\
\hline 38 & 77 & 93.506 & 4 & 1 & 7420 & 7495 & 131,293 & 131,217 & \\
\hline 39 & 56 & 100 & 0 & 0 & 87,421 & 87,476 & 346,975 & 346,920 & $r p / 2$ \\
\hline 40 & 56 & 100 & 0 & 0 & 155,027 & 155,082 & 346,920 & 346,975 & rps12(exon) \\
\hline 41 & 65 & 92.308 & 2 & 3 & 54,754 & 54,816 & 448,383 & 448,446 & atpE \\
\hline Total & 18,637 & & & & & & & & \\
\hline
\end{tabular}

genes account for only $4.31 \%$, which is probably the result of a gradual increase in sequence duplication during evolution. Most PCGs were the typical ATG start codon, and the distribution of amino acid compositions was similar to other angiosperms $[28,33]$. while the atp6, nad1 and
nad4L genes use ACG as initiation codons, this phenomenon also has been found in other studies, which were considered to be altered by RNA editing modification $[9,14]$. The cox 1 is one of the most reported mitochondrial genes involved in horizontal gene transfer among 


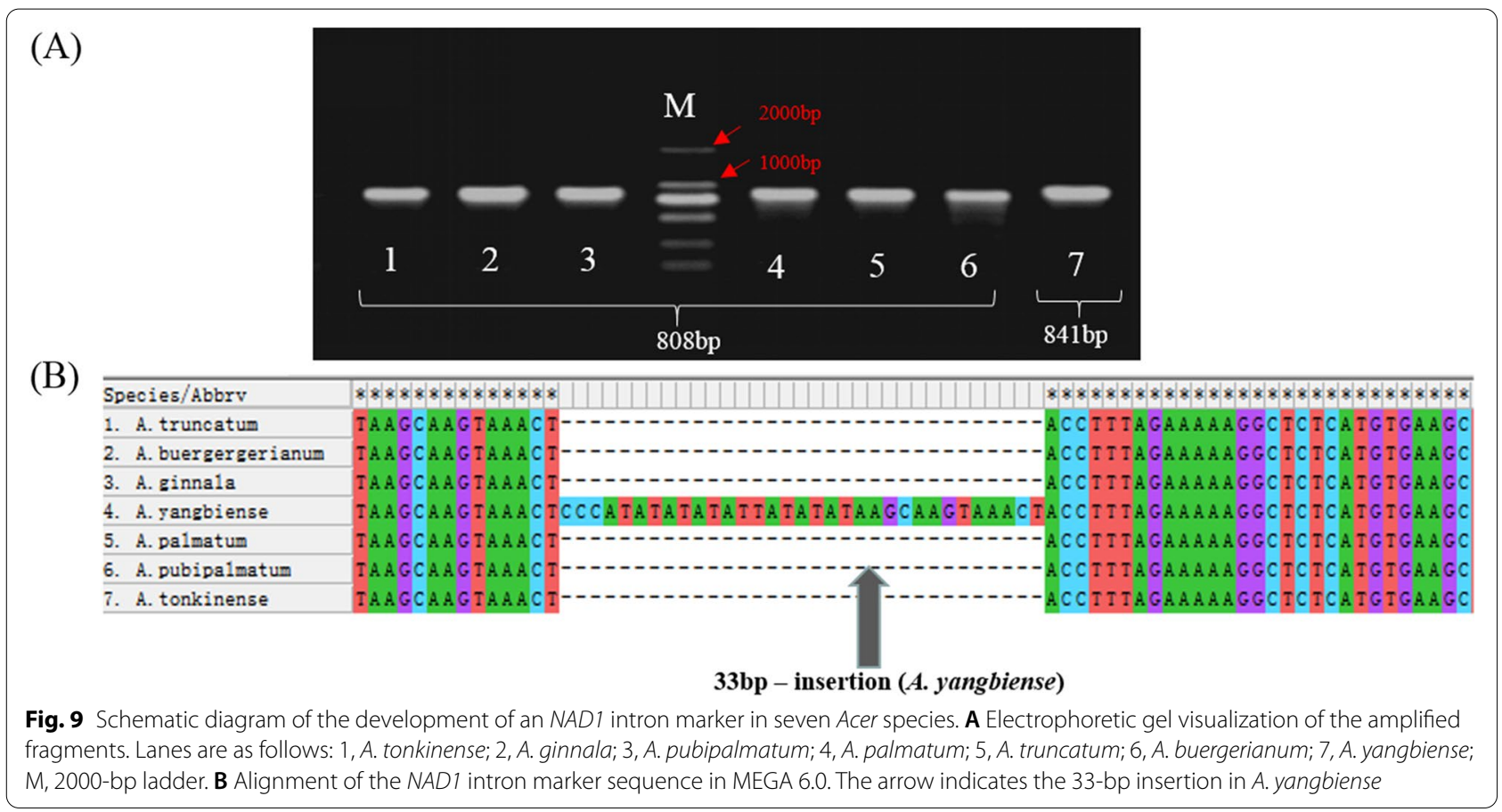

sngiosperms. In our study, two copies of $\operatorname{cox} 1$ genes were found. The previous studies reported that the cox 1 copies existed in different species and different populations of a species [35]. $\mathrm{Ka} / \mathrm{Ks}$ ratios $>1$ have also been reported for some other mitochondrial genes $[9,14,26]$. In our study, the high $\mathrm{Ka} / \mathrm{Ks}$ ratios of genes observed were very important for further studies in the gene selection and evolution of Acer species, including atp6, cob, cox1, nad2, ccmFn etc.

\section{Identification of repeat sequences and RNA editing sites}

Repeats are important sources of information for developing markers for population and evolutionary analyses $[23,36,37]$. Including tandem, short and large repeats, they are widely present in mitogenomes [14, 38, 39]. Repeats in mitochondrial DNA are generally vital for intermolecular recombination, which can generate structural variations and extreme mitogenome sizes [20,40]. In this study, five pair of large repeats $(>1 \mathrm{~kb})$ by rearrangements could produce two subgenomic circles in $A$. truncatum mitogenome, comprising of $457,840 \mathrm{bp}$ and $333,212 \mathrm{bp}$, respectively. This phenomenon also reported in Soybean [41]. we also found major differences between the repeat sequences of $A$. truncatum and $A$. yangbiense mitogenomes. In particular, the proportion of long repeat sequences in the $A$. truncatum mitogenome (18.24\%) was higher than that of $A$. yangbiense (17.20\%), and the longest repeats were $28,452 \mathrm{bp}$ and $27,124 \mathrm{bp}$, respectively. These repeats may have contributed to the increase in the mitogenome size of $A$. yangbiense. This finding also suggests that intermolecular recombination has frequently occurred in the mitogenome during Acer evolution [14, 31].

RNA editing, a post-transcriptional process that occurs in chloroplast and mitochondrial genomes of higher plants, contributes to improved protein folding $[9,14$, 26]. Previous researches had uncovered approximately 491 RNA editing sites within 34 genes in rice [42] and 486 RNA editing sites within 31 genes in P. vulgaris [14]. In the present study, we predicted RNA editing sites in 26 PCGs common to A. truncatum, A. yangbiense, A. thaliana and $C$. sinensis mitogenomes. We found that the number of RNA editing sites in PCGs was extremely conserved in Acer but differed in the other two species. Although the number of RNA editing sites varies greatly among genes, cytochrome $c$ biogenesis and NADH dehydrogenase genes harbor the largest number, which is similar to P. vulgaris [14]. In addition, all identified RNA editing sites are located at first and second codon positions. Previous researchers have speculated that the lack of RNA editing sites at the third codon position is probably due to the limitations of the PREP-Mt predictive methodology used rather than an actual absence [14, 43]. Further analysis using experimental methods is thus needed. 


\section{DNA fragment transfer events}

Information pertaining to DNA transfer events between different genomes (mitochondrial, nuclear and chloroplast) has been uncovered by sequencing analysis [21, 44, 45]. Previous studies have determined that the most prominent transfer direction in angiosperms is from organellar genomes into the nuclear genome, followed in importance by transfer from nuclear and plastid genomes into the mitogenome [13, 21, 46-48]. The total length of transferred DNA varies among plant species in higher plants, lengths range from $50 \mathrm{~kb}$ (A. thaliana) to $1.1 \mathrm{Mb}$ (O. sativa subsp. japonica) [49]. According to our study, $230.0 \mathrm{~kb}$ of nuclear DNA has been transferred into the mitogenome of $A$. truncatum. Although the nuclearmitochondrial transfer process has occurred on every $A$. truncatum chromosome, the total lengths of transferred material and the percent coverage differs among chromosomes. In total, $62,241 \mathrm{bp}$ of sequences $(7.87 \%$ of the $A$. truncatum mitogenome) is shared between nuclear and mitochondrial genomes. Most genes with transferred sequences shared between nuclear and mitochondrial genomes are tRNA genes, such as trnN-GTT, trnH-GTG , and trnH-GTG. Chang et al. [41] have reported similar results in soybean. In regards to chloroplast genome to mitogenome migration events, a total of $18,637 \mathrm{bp}$ of transferred fragments were observed, accounting for $2.36 \%$ of the A. truncatum mitogenome. In comparison, the proportion in S. suchowensis and Suaeda glauca is 2.8 and $5.18 \%$, respectively [31]. We identified 41 fragments that had been transferred from the chloroplast genome to the mitogenome, these fragments included six integrated genes, namely, five tRNA genes and $p s b J$. Transfer of tRNA genes from chloroplast to mitochondrial DNA is common in angiosperms [21, 26, 31]. Interestingly, we also observed that DNA migration often occurred in the inverted repeat region of the A. truncatum chloroplast genome.

\section{Development of a mitochondrial NAD1 intron marker for Acer species}

Because indel regions are relatively easy to detect, they are often used to develop markers for identifying species [50]. The genus Acer comprises more than 200 species grown in China $[2,51]$; however, the highly similar shapes of some species present a challenge for identification, and a molecular approach would be beneficial. $N A D 1$ intron indel markers have been useful for identification of some plant species [52-54]. In Acer, only the mitogenome of $A$. yangbiense has been previously reported [28]. In the present study, we first identified a 33-bp sequence difference by aligning the NAD1 intron regions of $A$. truncatum and $A$. yangbiense. Amplification of the NAD1 intron with specific primers revealed that a 33-bp indel was present in A. yangbiense, whereas the amplified NAD1 intron sequence was of the same length and highly conserved in the other six species. We verified that this 33-bp indel was an insertion in Acer by analyzing several species close to A. truncatum in our phylogenetic tree (A. yangbiense, $P$. tremula, S. suchowensis, and $C$. sinensis). The development of mitogenome-based molecular markers has not been previously reported for Acer. Although only a few Acer species were used in this study, our findings should nonetheless contribute to species classification in Acer.

\section{Conclusions}

In this study, we assembled and annotated the mitogenome of A. truncatum and performed extensive analyses based on DNA and amino acid sequences of annotated genes. The $A$. truncatum mitogenome is circular, with a length of 791,052 bp. We annotated 62 genes, including 35 protein-coding, 23 tRNA and 4 rRNA genes. In addition, the codon usage, sequence repeats, RNA editing and selective pressure were also analyzed in the $A$. truncatum mitogenome. The evolutionary status of $A$. truncatum was verified by phylogenetic analysis based on the mitogenomes of this species and 25 other taxa. Gene conservation between chloroplast and mitochondrial genomes and between nuclear and mitochondrial genomes were also detected in A. truncatum by analyzing gene migration. Finally, a newly developed NAD1 intron indel marker was used to distinguish Acer species. Our study has yielded extensive information about the $A$. truncatum mitogenome. The data presented herein supplement the genetic knowledge available for the genus Acer, provide novel insights into A. truncatum evolution, and form an important theoretical basis for increasing $A$. truncatum seed yield.

\section{Materials and methods Plant materials and DNA sequencing}

A. truncatum plants were grown at our Aceraceae seed base of Jangsu Academy of Agricultural Sciences (Lishui District, Nanjing, China; $31^{\circ} 65 \mathrm{~N}, 119^{\circ} 02 \mathrm{E}$ ) under natural conditions. Fresh leaves were frozen in liquid nitrogen and stored at $80^{\circ} \mathrm{C}$. DNA extraction and sequencing were performed using methods described in our previous de novo genome sequencing study [2].

\section{Mitogenome assembly and annotation}

For the A. truncatum mitogenome, PacBio RS II reads (59.42 GB) sequenced in our previous study [2] were de novo assembled using Canu v1.4 [55]. The obtained contigs were mapped to core mitochondrial genes by minimap2 [56], then extended. The assembled contigs were 
polished (Pilon v1.18) with Illumina reads (75.0 GB) to correct read errors [57]. Finally, five large contigs were assembled into mitogenome, to verify the quality and accuracy of our assemblies, we further verified the junctions by Sanger sequencing (Table S6 and Fig. S4). The GE-Seq tool on the MPI-MP CHLOROBOX website (https://chlorobox.mpimp-golm.mpg.de) was used for the mitogenome annotation, with the A. yangbiense mitogenome (CM017774.1) serving as a reference. Mitochondrial protein-coding genes were predicted using the MITOFY webserver [40]. All tRNA genes were confirmed by using tRNAscan-SE with default settings [9, 14, 58]. ORFfinder (https://www.ncbi.nlm.nih.gov/orffi nder/) was used to analyze open reading frames longer than $300 \mathrm{bp}$. RSCU values and the amino acid composition of PCGs were calculated in MEGA X [59]. A circular mitochondrial map was drawn using Organellar Genome DRAW [60].

\section{Analysis of repeat structures and SSRs}

Forward, reverse, palindromic and complementary repeats were identified with REPuter [61]. SSRs were analyzed with the MISA program [62]. The motif size of one- to six- nucleotide SSRs was set as 8, 4, 4, 3 and 3, respectively.

\section{Selective pressure analysis}

We calculated the nonsynonymous (Ka) and synonymous (Ks) substitution rates of each PCG between $A$. truncatum and A. yangbiense, A. thaliana, and C. sinensis. Orthologous gene pairs were separately aligned in MEGA 6.0. $\mathrm{Ka}$, Ks, and $\mathrm{Ka} / \mathrm{Ks}$ values were calculated using DnaSP [63].

\section{Genome alignments}

The $A$. truncatum mitogenome was searched against the chloroplast genome of A. truncatum (MH638284) using BLASTN 2.9.0+ according to the following screening criteria: matching rate $\geq 70 \%$, E-value $\leq 1 \mathrm{e}^{-6}$, and length $\geq 40$ [31]. To identify regions of potential nuclear origin in the mitogenome of $A$. truncatum, we also performed a BLASTN search (maximum E-value $=1 \mathrm{e}^{-50}$ ) of the complete mitogenome against all contigs from the A. truncatum nuclear genome sequenced in our previous study. BLASTN results of sequences longer than $250 \mathrm{bp}$ and a pairwise similarity $>80 \%$ were inspected for sequence features.

\section{Prediction of RNA editing sites}

RNA editing sites in the PCGs of $A$. truncatum and other three mitogenomes (A. yangbiense, A. thaliana and $C$. sinensis) were predicted using the the online PREP-Mt suite of servers (http://prep.unl.edu/). To obtain a more accurate prediction, the cutoff value was set as 0.2 [43]..

\section{Phylogenetic analyses}

A total of 26 complete mitogenomes (Table S4) were used to ascertain the phylogenetic position of $A$. truncatum. The 25 mitochondrial PCG genes (atp1, atp4, atp6, atp8, atp9, ccmB, ccmC, ccmFc, ccmFn, cob, cox1, cox3, matR, nad1, nad2, nad3, nad4, nad4L, nad5, nad6, nad7, nad 9, rps12, rps3, and rps4) conserved across the 26 analyzed species were aligned in Muscle with default parameters [64], with the alignment then modified manually to eliminate gaps and missing data. Finally, a maximum likelihood tree was constructed in MEGA $\mathrm{X}$ using the $\mathrm{JTT}+\mathrm{G}+\mathrm{I}+\mathrm{F}$ nucleotide substitution model [58]. A bootstrap consensus tree was inferred from 1000 bootstrap replicates. Triticum aestivum, Sorghum bicolor, Ginkgo biloba, and Zea mays were used as outgroups.

\section{Verification of the Nad1 insertion in Acer}

Primers were designed with Primer 5. PCR amplifications were carried out in $15-\mu$ l volumes containing $20 \mathrm{ng}$ genomic DNA, $0.4 \mu \mathrm{l}$ dNTPs $(2.5 \mathrm{mM}$ each), $2.5 \mu \mathrm{l}$ of $10 \times$ Ex Taq buffer $\left(\mathrm{Mg}^{2+}\right), 0.4 \mu \mathrm{l}$ Ex Taq DNA polymerase (Takara,Tokyo, Japan), and $1.0 \mu \mathrm{l}$ of each primer $(10 \mathrm{mM})$. The amplification conditions were $94{ }^{\circ} \mathrm{C}$ for $5 \mathrm{~min}$, followed by 30 cycles of $94{ }^{\circ} \mathrm{C}$ for $30 \mathrm{~s}, 56^{\circ} \mathrm{C}$ for $30 \mathrm{~s}$, and $72{ }^{\circ} \mathrm{C}$ for $30 \mathrm{~s}$, with a final extension of $72^{\circ} \mathrm{C}$ for $10 \mathrm{~min}$. The PCR products were purified and linked to the pMD19-T easy plasmid (Takara) for sequencing to confirm the accuracy of PCR product sizes. Three samples per species were sequenced by the General Biology Company (Nanjing, Jiangsu, China).

\section{Abbreviations}

Ile: Isoleucine; Leu: Leucine; Ser: Serine; PCR: Polymerase chain reaction; SSRs: Simple sequence repeat; PCG: Protein-coding genes; DNA: Deoxyribonucleic acid; SNPs: Single Nucleotide Polymorphism; tRNA: Tranfer RNA; rRNA: Ribosomal RNA; Arg: Arginine; Cys: Cysteine; Trp: Tryptophan; RSCU: Relative synonymous codon usage; $K a / K$ : Snonsynonymous-to-synonymous substitution ratio.

\section{Supplementary Information}

The online version contains supplementary material available at https://doi. org/10.1186/s12870-021-03416-5.

Additional file 1: Figure S1. Reversible reorganization of the A. truncatum mitgenome may produce subgenomic circles by large repeats. The same colour triangles represent the pairs of large repeats.

Additional file 2: Figure S2. The type of detected repeats and the frequency distribution of lengths in the $A$. yangbiense.

Additional file 3: Figure S3. Alignment of the NAD1 intron sequence with MEGA-X. 
Additional file 4: Figure S4. Agarose gel electrophoresis of PCR product for contig connecting verification

Additional file 5: Table S1. The repeat sequences distributions in the A. truncatum mitogenome genome.

Additional file 6: Table S2. The large repeats ( $>1 \mathrm{~kb}$ ) by rearrangements could produce two subgenomic circles in A. truncatum mitogenome.

Additional file 7: Table S3. The repeat sequences distributions in the A. yangbiense mitogenome genome.

Additional file 8: Table S4. Details regarding the mitochondira genome sequences used for the phylogenetic analysis.

Additional file 9: Table S5. Details regarding the primers used to develop the NAD1 intron marker.

Additional file 10: Table S6. Primers for contig connecting verification

\section{Acknowledgments}

Thanks to all the members of the Institute of Leisure Agriculture team for their contributions, and the Mitogenome assembly and annotation by the Genepioneer Biotechnologies Co, Ltd.

\section{Authors' contributions}

QYM, QZL, CWB designed the project and the strategy, SXL, JW, ZC and YMD contributed to plant sample collection; QYM, YXW, CWB, LZ, JR and KYY work on genome assembly, annotation and comparative analyses; QYM, CWB and QZL wrote and revised the manuscript. All authors read and approved the final manuscript.

\section{Funding}

This work was funded by the Natural Science Foundation of China (32001357), Natural Science Foundation of Jiangsu Province (BK20211139), the Independent Innovation Fund Project of Agricultural Science and Technology in Jiangsu Province (CX (20)3155).

\section{Availability of data and materials}

The A. truncatum Mitochondrial genome sequence was deposited in the GenBank database (accession number MZ318049).

\section{Declaration}

\section{Competing interests}

The authors declare that they have no conflicts of interest.

\section{Author details}

'Institute of Leisure Agriculture, Jiangsu Academy of Agricultural Sciences, Nanjing 210014, China. ${ }^{2}$ Nanjing Forestry University, Nanjing 210037, China. ${ }^{3}$ Institute of Agricultural Engineering, Anhui Academy of Agricultural Sciences, 40 Nongkenanlu, Hefei 230031, Anhui, China.

\section{Received: 14 August 2021 Accepted: 27 December 2021}

Published online: 13 January 2022

\section{References}

1. Guo X, Wang R, Chang R, Liang X, Wang C, Luo Y, et al. Effects of nitrogen addition on growth and photosynthetic characteristics of Acer truncatum seedlings. Dendrobiology. 2014;72:151-61. https://doi.org/10.12657/ denbio.072.013.

2. Ma Q, Sun T, Li S, Wen J, Zhu L, Yin T, et al. The Acer truncatum genome provides insights into nervonic acid biosynthesis. Plant J. 2020;104(3):662-78. https://doi.org/10.1111/tpj.14954.

3. Tang W, Wang J, Xu J, Wang L, Huang J, Chen Y. Advances of chemical composition of medicinal plants in Aceraceae. Northern Horticulture. 2012;18:194-200

4. Wang $X$, Wang S. A new resource of nervonic acid from purpleblow maple (Acer truncatum) seed oil. For Prod J. 2005;09:60-2.
5. Tanaka K, Shimizu T, Ohtsuka Y, Yamashiro Y, Oshida K. Early dietary treatments with Lorenzo's oil and docosahexaenoic acid for neurological development in a case with Zellweger syndrome. Brain and Development. 2007;29:586-9. https://doi.org/10.1016/j.braindev.2007.02.005.

6. Amminger G, Schäfer M, Klier C, Slavik J, Holzer I, Holub M. Decreased nervonic acid levels in erythrocyte membranes predict psychosis in help-seeking ultra-high-risk individuals. Mol Psychiatry. 2012;17:1150-67. https://doi.org/10.1038/mp.2011.167.

7. Taylor D, Francis T, Guo Y, Brost J, Katavic V, Mietkiewska E. Molecular cloning and characterization of a KCS gene from Cardamine graeca and its heterologous expression in Bracssica oilseeds to engineer high nervonic acid oils for potential medical and industrial use. Plant Biotechnol J. 2009;7:925-38. https://doi.org/10.1111/j.1467-7652.2009.00454.X.

8. Guo Y, Mietkiewska E, Francis T, Katavic V, Brost J, Giblin M. Increase in nervonic acid content in transformed yeast and transgenic plants by introduction of a Lunaria annua L. 3-ketoacyl- CoA synthase (KCS) gene. Plant Mol Biol. 2009;69:565-75. https://doi.org/10.1007/s11103-008-9439-9.

9. Ye N, Wang X, Li J, Bi C. Assembly and comparative analysis of complete mitochondrial genome sequence of an economic plant Salix suchowensis. Peer J. 2017;5(1):e3148. https://doi.org/10.7717/peerj.3148.

10. Birky C. Uniparental inheritance of mitochondrial and chloroplast genes: mechanisms and evolution. Proc Nati Acad Sci. 1995;92:11331-8.

11. Cusimano N, Wicke S. Massive intracellular gene transfer during plastid genome reduction in nongreen Orobanchaceae. New Phytol. 2016;210(2):680-93. https://doi.org/10.1111/nph.13784.

12. Bock R. Witnessing genome evolution: experimental reconstruction of endosymbiotic and horizontal gene transfer. Annu Rev Genet. 2017;51(1):1-22. https://doi.org/10.1146/annurev-genet-120215-035329.

13. Zhao N, Grover C, Chen Z, Wendel J, Hua J. Intergenomic gene transfer in diploid and allopolyploid Gossypium. BMC Plant Biol. 2019;19(1):492. https://doi.org/10.1186/s12870-019-2041-2.

14. Bi C, Lu N, XuY, He C. Characterization and analysis of the mitochondrial genome of common bean (Phaseolus vulgaris) by comparative genomic approaches. Int J Mol Sci. 2020;21(11):3778. https://doi.org/10.3390/ijms2 1113778.

15. Hsu C, Mullin B. Physical characterization of mitochondrial DNA from cotton. Plant Mol Biol. 1989;13:467-8. https://doi.org/10.1007/BF00015558.

16. Greiner $S, B o c k$ R. Tuning a ménage à trois: co-evolution and co-adaptation of nuclear and organellar genomes in plants. Drug Alcohol Depend. 2013;35:354-65. https://doi.org/10.1002/bies.201200137.

17. Richardson A, Rice D, Young G, Alverson A, Palmer J. The, "Fossilized" mitochondrial genome of Liriodendron tulipifera: ancestral gene content and order, ancestral editing sites, and extraordinarily low mutation rate. BMC Biol. 2013;11:29. https://doi.org/10.1186/1741-7007-11-29.

18. Skippington E, Barkman T, Rice D, Palmer J. Miniaturized mitogenome of the parasitic plant Viscum scurruloideum is extremely divergent and dynamic and has lost all nad genes. Proc Natl Acad Sci U S A. 2015;112(27):E3515. https://doi.org/10.1073/pnas.1504491112.

19. Sloan D, Alverson A, Chuckalovcak J, Wu M, Mccauley D. Rapid evolution of enormous, multichromosomal genomes in flowering plant mitochondria with exceptionally high mutation rates. PLoS Biol. 2012;10(1):e1001241. https://doi.org/10.1371/journal.pbio.1001241.

20. Guo W, Felix G, Fan W, Young G. Ginkgo and Welwitschia Mitogenomes reveal extreme contrasts in gymnosperm mitochondrial evolution. Mol Biol Evol. 2016;33(6):1448-60. https://doi.org/10.1093/molbev/msw024.

21. Bergthorsson U, Adams K, Thomason B, Palmer J. Widespread horizontal transfer of mitochondrial genes in flowering plants. Nature. 2003;424(6945):197-201. https://doi.org/10.1038/nature01743.

22. Wynn E, Christensen A. Repeats of unusual size in plant mitochondrial genomes: identification, incidence and evolution. G3: genes, genomes. Genetics. 2019;9:549-59. https://doi.org/10.1534/g3.118.200948.

23. Ma Q, Li S, Bi C, Hao Z, Sun C, Ye N. Complete chloroplast genome sequence of a major economic species, Ziziphus jujuba (Rhamnaceae). Curr Genet. 2017;63:117-29. https://doi.org/10.1007/s00294-016-0612-4.

24. Seok J, Kim A, Wang J, Hee K, Iksoo L. Single-nucleotide polymorphism markers in mitochondrial genomes for identifying Varroa destructorresistant and -susceptible strains of Apis mellifera (Hymenoptera: Apidae). Mitochondrial DNA Part A, DNA Mapp Seq Anal. 2019;30(3):477-89. https://doi.org/10.1080/24701394.2018.1551385.

25. Mwamuye M, Obara I, Khawla E, Odongo D, Bakheit M, Jongejan F, et al. Unique mitochondrial single nucleotide polymorphisms demonstrate 
resolution potential to discriminate Theileria parva vaccine and Buffaloderived strains. Life. 2020;10:334. https://doi.org/10.3390/life10120334.

26. Bi C, Paterson A, Wang X, Xu Y, Wu D, Qu Y, et al. Analysis of the complete mitochondrial genome sequence of the diploid cotton Gossypium raimondii by comparative genomics approaches. Biomed Res Int. 2016;2016:5040598. https://doi.org/10.1155/2016/5040598.

27. Choi I, Schwarz E, Ruhlman T, Khiyami M, Sabir J, Hajarah N, et al. Fluctuations in Fabaceae mitochondrial genome size and content are both ancient and recent. BMC Plant Biol. 2019;19(1):448. https://doi.org/10. 1186/s12870-019-2064-8

28. Yang J, Wariss H, Tao L, Zhang R, Yun Q, Hollingsworth P, et al. De novo genome assembly of the endangered Acer yangbiense, a plant species with extremely small populations endemic to Yunnan Province, China. Gigascience. 2019;8(7):giz085. https://doi.org/10.1093/gigascience/ giz085.

29. Ma Q, Wang Y, Zhu L, Bi C, Li S, Li S, et al. Characterization of the complete chloroplast genome of Acer truncatum bunge (Sapindales: Aceraceae): a new woody oil tree species producing nervonic acid. Biomed Res Int. 2019;11:1-13. https://doi.org/10.1155/2019/7417239.

30. Kozik A, Rowan B, Lavelle D, Berke L, Schranz M, Michelmore R, et al. The alternative reality of plant mitochondrial DNA: one ring does not rule them all. PLoS Genet. 2019;15(8):e1008373. https://doi.org/10.1371/journ al.pgen.1008373.

31. Cheng $Y$, He $X$, Priyadarshani $S$, Wang $Y$, Ye L, Qin Y, et al. Assembly and comparative analysis of the complete mitochondrial genome of Suaeda glauca. BMC Genomics. 2021;22:167. https://doi.org/10.1186/ s12864-021-07490-9.

32. Chang S, Yang T, Du T, Huang Y, Chen J, et al. Mitochondrial genome sequencing helps show the evolutionary mechanism of mitochondrial genome formation in Brassica. BMC Genomics. 2021;12:497. https://doi. org/10.1186/1471-2164-12-497.

33. Sloan D, Wu Z, Sharbrough J. Correction of persistent errors in Arabidopsis reference mitochondrial genomes. Plant Cell. 2018;30(3):525-7. https:// doi.org/10.1105/tpc.18.00024.

34. Kubo T, Nishizawa S, Sugawara A, Itchoda N, Estiati A, Mikami T. The complete nucleotide sequence of the mitochondrial genome of sugar beet (Beta vulgaris L.) reveals a novel gene for tRNACys (GCA). Nucleic Acids Res. 2000;28:2571-6. https://doi.org/10.1093/nar/28.13.2571.

35. Zhang C, Ma H, Sanchez-Puerta MV, Li L, Xiao J, et al. Horizontal gene transfer has impacted cox 1 gene evolution in Cassytha filiformis. J Mo Evol. 2020;88(4):361-71. https://doi.org/10.1007/s00239-020-09937-1.

36. Xiong Y, Lei X, Bai S, Xiong Y, Liu W, Wu W, et al. Genomic survey sequencing, development and characterization of single- and multi-locus genomic SSR markers of Elymus sibiricus L. BMC Plant Biol. 2021;21:3. https://doi.org/10.1186/s12870-020-02770-0.

37. Liu L, Fan X, Tan P, Wu J, Zhang H, Han C, et al. The development of SSR markers based on RNA-sequencing and its validation between and within Carex L. species. BMC Plant Biol. 2021;21:17. https://doi.org/10. 1186/s12870-020-02792-8.

38. Gualberto J, Mileshina D, Wallet C, Niazi A, Weber-Lotfi F, Dietrich A. The plant mitochondrial genome: dynamics and maintenance. Biochimie. 2014;100:107-20. https://doi.org/10.1016/j.biochi.2013.09.016.

39. Guo W, Zhu A, Fan W, Mower J. Complete mitochondrial genomes from the ferns Ophioglossum californicum and Psilotum nudum are highly repetitive with the largest organellar introns. New Phytol. 2017;213(1):391-403. https://doi.org/10.1111/nph.14135.

40. Alverson AJ, et al. Insights into the evolution of mitochondrial genome size from complete sequences of Citrullus lanatus and Cucurbita pepo (Cucurbitaceae). Mol Biol Evol. 2010;27(6):1436-48. https://doi.org/10. 1093/molbev/msq029.

41. Chang S, Wang Y, Lu J, Gai J, Li J, Chu P, et al. The mitochondrial genome of soybean reveals complex genome structures and gene evolution at intercellular and phylogenetic levels. PLoS One. 2031;8(2):e56502. doi. https://doi.org/10.1371/journal.pone.0056502.

42. Notsu Y, Masood S, Nishikawa T, Kubo N, Akiduki G, Nakazono M, et al. The complete sequence of the rice (Oryza sativa L.) mitochondrial genome: frequent DNA sequence acquisition and loss during the evolution of flowering plants. Mol gen. Genomics. 2002;268(4):434-45. https://doi. org/10.1007/s00438-002-0767-1.

43. Mower J. PREP-Mt: predictive RNA editor for plant mitochondrial genes. BMC Bioinformatics. 2005;6:96. https://doi.org/10.1186/1471-2105-6-96.
44. Timmis J, Ayliffe M, Huang C, Martin W. Endosymbiotic gene transfer: organelle genomes forge eukaryotic chromosomes. Nat Rev Genet. 2004;5:123-35. https://doi.org/10.1038/nrg1271.

45. Nguyen V, Giang V, Waminal N, Park H, Kim N, Jang W, et al. Comprehensive comparative analysis of chloroplast genomes from seven Panax species and development of an authentication system based on species-unique single nucleotide polymorphism markers. J Ginseng Res. 2000;44:135-44. https://doi.org/10.1016/j.jgr.2018.06.003.

46. Martin W, Stoebe B, Goremykin V, Hansmann S, Hasegawa M, Kowallik KV. Gene transfer to the nucleus and the evolution of chloroplasts. Nature. 1998;393(6681):162-5. https://doi.org/10.1038/30234.

47. Zhao N, Wang Y, Hua J. The roles of mitochondrion in intergenomic gene transfer in plants: a source and a pool. Int J Mol Sci. 2018;19(2):E547. https://doi.org/10.3390/ijms19020547.

48. Rice D, Alverson A, Richardson A, Young G, Sanchez-Puerta M, Munzinger $J$, et al. Horizontal transfer of entire genomes via mitochondrial fusion in the angiosperm Amborella. Science. 2013;342(6165):1468-73. https://doi. org/10.1126/science.1246275.

49. Smith D, Crosby K, Lee R. Correlation between nuclear plastid DNA abundance and plastid number supports the limited transfer window hypothesis. Genome Biol Evol. 2011;3:365-71. https://doi.org/10.1093/ gbe/evr001.

50. Sebbenn A, Blanc-Jolivet C, Mader M, Meyer-Sand B, Degen B. Nuclear and plastidial snp and indel markers for genetic tracking studies of jacaranda copaia. Conservation Genet Resour. 2019;2019(11):341-3. https:// doi.org/10.1007/s12686-019-01097-9.

51. Xu T. The systematic evolution and distribution of the genus Acer: Acta Botanica Yunnanica; 1998.

52. Chase F, Freudenstein J. Chase analysis of mitochondrial nad $1 \mathrm{~b}$-c intron sequences in Orchidaceae: utility and coding of length-change characters. Syst Bot. 2001;26(3):643-57. https://doi.org/10.1043/0363-6445-26.3. 643.

53. Zhang T, Wang Z, Xu L, Zhou K. Application of mitochondrial nad 1 intron 2 sequences to molecular identification of some species of dendrobium Sw. Chinese Traditional and Herbal Drugs. 2005;36(7):1059-62.

54. Pan Z, Ren X, Zhao H, Liu L, Tan Z, Qiu F. A mitochondrial transcription termination factor, $\mathrm{zmsmk} 3$, is required for nad 1 intron 4 and nad 4 intron 1 splicing and kernel development in maize. G3: Genes Genomes. Genetics. 2019;9(8):2677-86. https://doi.org/10.1534/g3.119.400265.

55. Koren S, Walenz B, Berlin K, Miller J, Phillippy A. Canu: scalable and accurate long-read assembly via adaptive $k$-mer weighting and repeat separation. Genome Res. 2017;27:722-36. https://doi.org/10.1101/gr. 215087.116.

56. Li H. Minimap2: pairwise alignment for nucleotide sequences. Bioinformatics. 2018;34(18):3094-100. https://doi.org/10.1093/bioinformatics/ bty191.

57. Walker B, Abeel T, Shea T, Priest M, Abouelliel A, Sakthikumar S, et al. Pilon: an integrated tool for comprehensive microbial variant detection and genome assembly improvement. PLoS One. 2014;9(11):e112963. https:// doi.org/10.1371/journal.pone.0112963.

58. Lowe T, Eddy S. tRNAscan-SE: a programfor improved detection of transfer RNA genes in genomic sequence. Nucleic Acids Res. 1997;25(5):95564. https://doi.org/10.1093/nar/25.5.955.

59. Kumar S, Stecher G, Li M, Knyaz C, Tamura K. MEGA X: molecular evolutionary genetics analysis across computing platforms. Mol Biol Evol. 2018;35(6):1547-9. https://doi.org/10.1093/molbev/msy096.

60. Greiner S, Lehwark P, Bock R. OrganellarGenomeDRAW (OGDRAW) version 1.3.1: expanded toolkit for the graphical visualization of organellar genomes. Nucleic Acids Res. 2019;47(W1):W59-64. https://doi.org/10. $1101 / 545509$

61. Kurtz S, Choudhuri J, Enno O, Chris S, Jens S, Robert G. REPuter: the manifold applications of repeat analysis on a genomic scale. Nucleic Acids Res. 2001;29(22):4633-42. https://doi.org/10.1093/nar/29.22.4633.

62. Thiel T, Michalek W, Varshney R, Graner A. Exploiting EST databases for the development and characterization of gene-derived SSR-markers in barley (Hordeum vulgare L.). Theor Appl Genet. 2003;106:411-22. https://doi.org/ 10.1007/s00122-002-1031-0

63. Librado RJ. DnaSP v5: a software for comprehensive analysis of DNA polymorphism data. Bioinformatics. 2009;25(11):1451-2. https://doi.org/ 10.1093/bioinformatics/btp187. 
64. Edgar R. MUSCLE: multiple sequence alignment with high accuracy and high throughput. Nucleic Acids Res. 2004;32(5):1792-7. https://doi.org/ 10.1093/nar/gkh340.

\section{Publisher's Note}

Springer Nature remains neutral with regard to jurisdictional claims in published maps and institutional affiliations.

- fast, convenient online submission

- thorough peer review by experienced researchers in your field

- rapid publication on acceptance

- support for research data, including large and complex data types

- gold Open Access which fosters wider collaboration and increased citations

- maximum visibility for your research: over 100M website views per year

At BMC, research is always in progress.

Learn more biomedcentral.com/submissions 